\section{THE LIQUID STATE}

$\mathrm{T}$ HE twenty-eighth Guthrie Lecture of the Physical Society was delivered last April by Dr. J. H. Hildebrand, professor of inorganic chemistry in the University of California. $\mathrm{He}$ had chosen for his subject "The Liquid State". Prof. Hildebrand is one of the greatest authorities on the physical and chemical properties of solutions, and it is only natural that his vast knowledge and experience in this field have enabled him to tackle the difficult problem of the constitution of liquids on new and promising lines.

Prof. Hildebrand began by outlining and discussing the two conventional methods by which the problem is usually approached: the analogies to the gaseous and to the solid state. A liquid resembles to some extent a compressed gas, and the theoretical treatment of such a model has the van der Waals equation as its basis. However, when we compare the terms of the van der Waals equation with their counterparts in a purely thermodynamic equation of state, we find that it is not possible to fit them with the same constants over any considerable range in the case of liquids. It is, of course, possible to correct the equation by additional constants, but this does not bring us nearer to the desired theoretical interpretation. The customary analogy between the behaviour of gases and liquids as represented by van't Hoff's law for osmotic pressure leads to impossible consequences when concentrated solutions are considered. Instead of describing a liquid as a compressed gas, it can be treated-starting from the other extreme-as a very disordered solid, that is, as a crystal lattice which has been disturbed by the process of melting. This method has been helped to a great extent by the considerable amount of information on the structure of solids at our disposal, and it is a line of approach which offers good prospects for a theoretical interpretation of the liquid state.

Quite a different line of approach can, however, be developed by using a method of interpretation which arises out of the experimental study with the aid of $\mathrm{X}$-rays, and it is on considerations of this kind that Prof. Hildebrand has based his views on the constitution of liquids. The scatter of X-rays in a liquid does not suggest a state of order in any way like that exhibited by a crystal lattice. Its interpretation reveals a short-range order which can be best represented by plotting the average number of molecular centres to be found in the neighbourhood of any given molecule against the distance from this molecule. The number of molecular centres contained in a spherical shell which surrounds the central molecule is given by the volume of the shell, multiplied by the number of molecular centres per unit volume of the substance. This estimate takes no account of the space required by the individual molecules, in so far as it clearly cannot be true for a shell of the order of magnitude of the minimum distance to which two molecules can approach. We must, therefore, introduce a correcting factor which varies with the distance from the central molecule and represents the structure of the liquid. This 'distribution function', $W$, is zero for any distance smaller than the molecular diameter. Beyond this distance we meet the first layer of surrounding molecules and $W$ will be greater than unity. Between the first and the second layer of surrounding molecules, $W$ shows a minimum, rising to a second maximum at the distance of the second surrounding layer. The success of this approach to a general interpretation of the structure

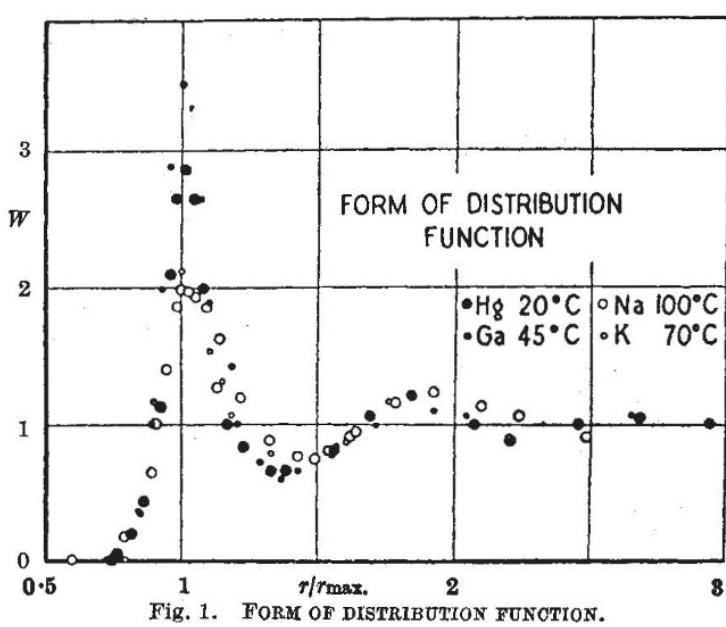

of liquids can be gathered from Fig. 1, which shows the distribution function for a number of liquid metals at corresponding states. Here $W$ is plotted against $r / r_{\max }$, where $r_{\max }$. is the position of the first maximum. The figure emphasizes the short range of order encountered in liquids, $W$ remaining practically 1 for distances greater than the radius of the second surrounding shell of particles.

The full significance of this treatment becomes apparent when we consider the forms which $W$ assumes in a solid and in a gas. In the latter case, $W$ rises from zero for distances smaller than that given by the closest approach at collision to the value of 1 , which is maintained for all greater distances. In the solid, the long-range order is represented in $W$ by a succession of tall, narrow bands which occur at distances where new groups of molecules will be found. The temperature dependency of the structure becomes apparent by a widening of these bands

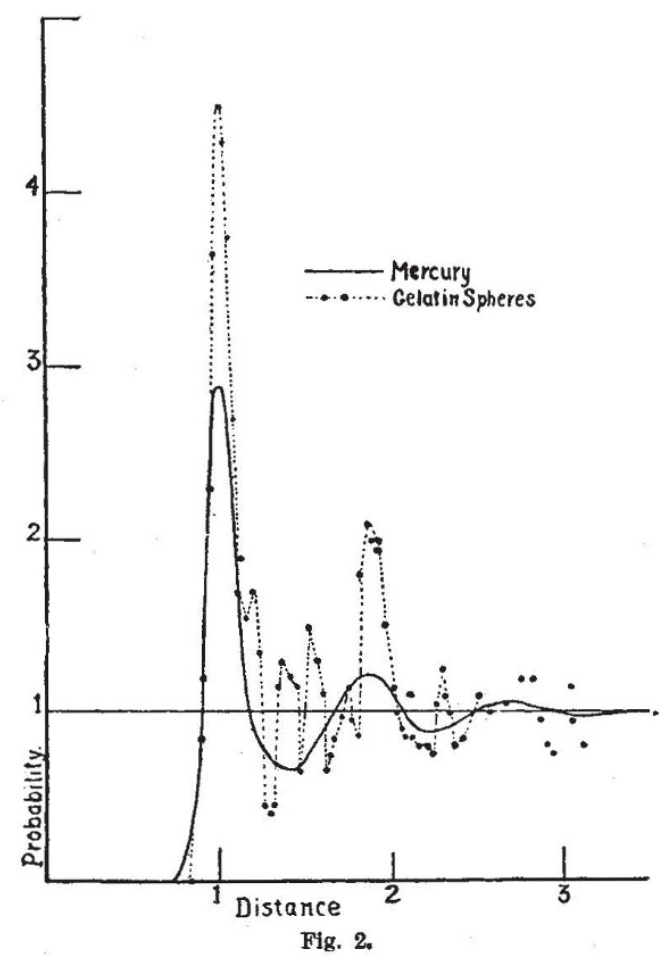




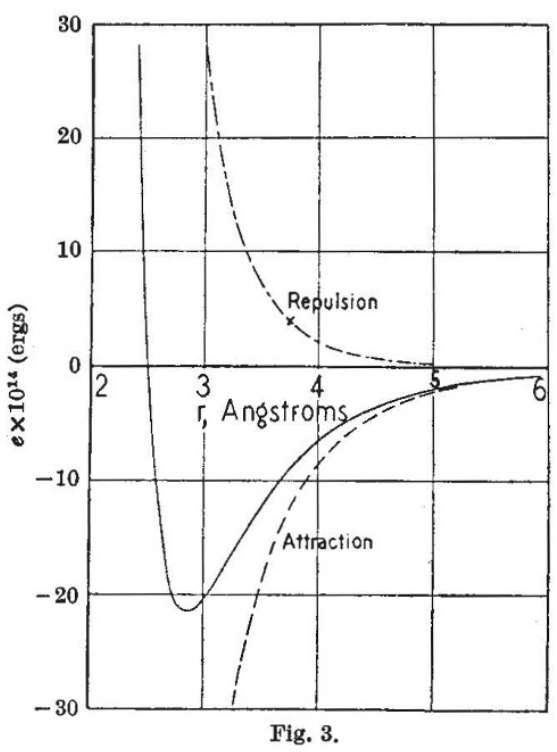

caused by the increasing amplitude of vibration of the molecular centres around the lattice points. At the melting-point the bands merge into the 'liquid' curve. With further rise in temperature the state of order in the liquid decreases progressively, the liquid curve flattens more and more, until finally $W$ is unity for any distance larger than the molecular diameter; the gaseous state has been reached. Prof. Hildebrand mentioned an ingenious demonstration experiment on a mechanical model of a liquid which had been carried out at his laboratory. The place of the molecules was taken by a number of gelatine spheres which were inserted in a cubical container, filled with a gelatine solution of the same refractive index as that of the spheres. This made the spheres invisible except for a few which had been coloured. The container was shaken, and the position of the coloured spheres determined by spark photographs. The $W$-function for this system was calculated from the results of these photographs and is reproduced in Fig. 2. For comparison the $W$ function for mercury is superposed.

By combining the known values of the heat of vaporization at different temperatures with the $W$ function, the potential between a pair of molecules of the liquid can be obtained. Splitting the potential into an attractive term $k / r^{6}$ and a repulsive potential $j / r^{n}$, the values for $k, j$ and $n$ can be found and the potential function constructed. This has actually been done from $\mathbf{X}$-ray results for mereury and the function obtained is given in Fig. 3. A test for the legitimacy of the method was provided by an independent calculation of $k$, which came to $3.35 \times 10^{-10}$, a value which is in excellent agreement with that of $3.52 \times 10^{-10}$ from the computation based on the $W$-function.

So far, only molecules have been considered which exhibit spherical symmetry in shape as well as in the field of force. There exists an equal maximum state of disorder in all liquids which are composed of spherical molecules, and on passing into the gaseous state they all must suffer the same increase in entropy on evaporation. This is simply Trouton's rule, which holds for all 'normal liquids', and which states that they all have equal entropies of vaporization at the boiling point, or, as Prof. Hildebrand would rather say, at the temperature where the molal volumes of vapour are equal. If, on the other hand, we deal with liquids the molecules of which are very elongated or contain dipoles, we can expect a certain degree of order in the spatial arrangement of these molecules, and the entropy of such liquids will be smaller than that of the completely disordered normal liquids. Since the entropies of all vapours under corresponding conditions are equal, the entropy of vaporization of liquids with non-spherical molecules will be greater than of normal ones. The significance of these exceptions from Trouton's rule is exemplified by the follow. ing table, in which the entropy of vaporization at equal molal vapour volumes $(\log R / V=0 \cdot 1)$ is given for a number of liquids.

\begin{tabular}{|c|c|c|}
\hline Liquid & $\Delta S$ & $\Delta S-20 \cdot 1$ \\
\hline 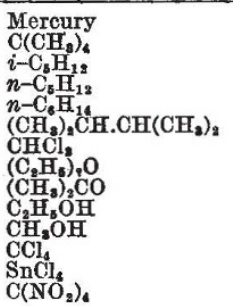 & $\begin{array}{l}20 \cdot 1 \\
20 \cdot 1 \\
20 \cdot 2 \\
20 \cdot 7 \\
21 \cdot 5 \\
20 \cdot 3 \\
21 \cdot 7 \\
21 \cdot 8 \\
22 \cdot 5 \\
27 \cdot 0 \\
26 \cdot 5 \\
20 \cdot 7 \\
21 \cdot 8 \\
22 \cdot 6\end{array}$ & $\begin{array}{l}0 \cdot 0 \\
0 \cdot 0 \\
0 \cdot 1 \\
0 \cdot 6 \\
1 \cdot 4 \\
0 \cdot 2 \\
1.6 \\
1.7 \\
2 \cdot 4 \\
6.9 \\
6 \cdot 4 \\
0 \cdot 6 \\
1 \cdot 7 \\
2 \cdot 5\end{array}$ \\
\hline
\end{tabular}

The second column, giving the differences between the vaporization entropies of the various liquids and that of mercury, denotes their state of order compared with the maximum disorder for spherical molecules. The degree of order which is introduced by alterations in the shape of the molecule is apparent in the differences between the pentanes, between di-isopropyl and normal hexane, and finally in the series carbon tetrachloride, stannic chloride and tetra-nitromethane. The deviations from Trouton's rule shown by chloroform, ether and acetone are due to the existence of a strong dipole in the molecule, and to the order created by the mutual interaction of these dipoles in the liquid. It is interesting to see that, though they all have dipole moments of roughly equal strength, the state of order in acetone is considerably greater than in the other two. The explanation is that the dipole in acetone is not buried inside the molecule as in chloroform and ether. The most striking exception, however, is that of the alcohols, and it seems difficult to account for this large deviation by either of the explanations. More information on this point can be gained by dissolving these dipole molecules in non-polar solvents and by observing the change in polarization with concentration. The very strong rise with increasing concentration in the case of the alcohols suggests that the high degree of order in the pure liquid is probably due to the formation of hydrogen bridges.

This is just one example in which the study of solutions aids in the interpretation of the general problem of the constitution of liquids. Thus we can combine observations of the composition of solutions with observations of the vapour phase, which leads us to consider deviations from Raoult's law. This rule states that for a great number of solutions, ideal solutions as they are called, the partial vapour pressure of each component is simply given by the product of its vapour pressure in the pure state and its molar fraction in the solution. Reverting to a description in terms of molecular characteristics, it 


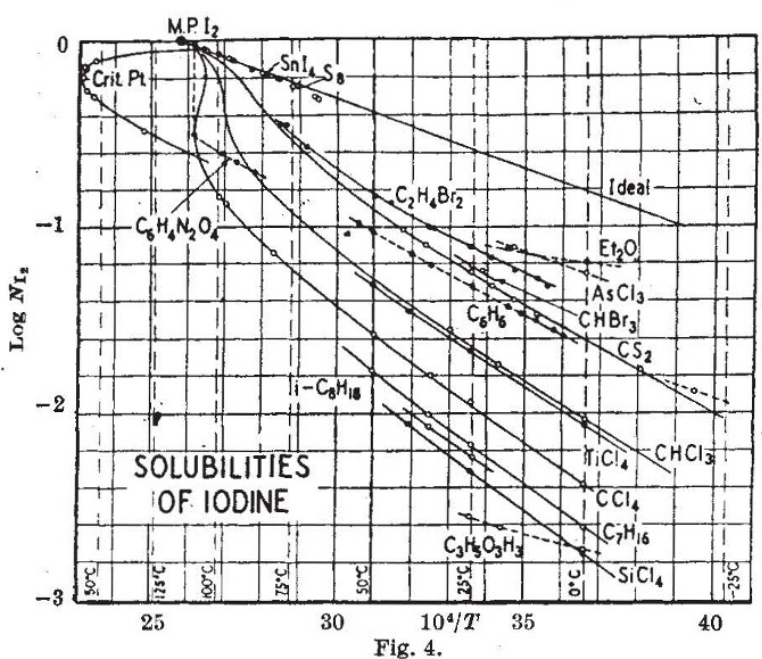

means that Raoult's law will hold for mixtures of the same species of molecule, that is, for molecules of equal size, shape and field of force. We can vary this condition by mixing different species and comparing the partial pressures. An interesting case is a mixture of molecules of equal field-strength, but of different size. Here the conceptions of the osmotic school, expressing concentrations in moles per litre, break down. According to these conceptions, we should expect, for example, ethane under one atmosphere to dissolve to the same extent in a given volume of any higher paraffin. On the other hand, the mole fraction of a given amount of ethane in dodecane would be twice that in the same volume of hexane. To achieve the same solubility the pressure of ethane over dodecane would have to be doubled. It is the latter alternative that is supported by the experiment. Normal paraffins of different length such as butane and heptane obey Raoult's law. It simply means that only the space between the molecules of the solvent is at the disposal of the second component, and not the total volume.

The properties of solutions offer almost unlimited scope for obtaining information on the problems of the liquid state. The same considerations as applied to the evaluation of the potential between a pair of identical molecules can be employed in evaluating potentials between molecules of different kind. If we have spherical molecules of equal size, but different molecular fields, the $W$-function is the same as in the pure liquid and can be made the base of calculations. Of the great number of examples given by Prof. Hildebrand, the set dealing with a comparison of different solvents for iodine is perhaps the most instructive one. The solubilities for this substance are given in Fig. 4. Most of the curves belong to one family; they represent 'regular solutions' for which computation on the lines indicated above is possible. They all show the violet colour of iodine vapour, which indicates that the iodine molecules are simply dissolved and have not taken part in chemical changes. The solutions corresponding to the non-regular curves, on the other hand, have different colours and make one suspect that chemical changes have occurred. Prof. Hildebrand directed special attention to the loop intersecting the curve for carbon tetrachloride. Calculation shows that the curve running through the points for the solubility of solid iodine must exhibit an S-shape, which means that there are two composi- tions in equilibrium at the same temperature. Experiments which had to overcome the difficulty that iodine solutions of such high concentration are quite opaque gave the completeliquid-liquid solubility curve in close approximation with the calculated values.

Results such as this show how far theoretical interpretation of the liquid state can provide an explanation of the observed phenomena, and can even be used in the prediction of conditions of considerable complexity. In his conclusion, Prof. Hildebrand left no doubt, however, that while there are remarkable achievements already at hand, the liquid state still abounds in unexplained phenomena which await elucidation when scientific men can return to its problems after the War. K. Mendelssonn.

\section{NUTRITION AND A MATTER OF TASTE}

\section{BY DR. MAGNUS PYKE}

$\mathrm{D}$ URING the past twenty-five or thirty years the science of nutrition has made very great strides. These advances have been due almost entirely to the application of precise, objective, chemical methods, and have been paralleled by similar advances in other branches of biology. Those who study endocrine secretions can determine, according to his endocrine balance, how the character of a man will be in. fluenced. Similarly, the nutritionist can say from an analysis of the foodstuffs of which a man makes up his diet whether or not, and in what way, his body will be influenced for good or evil. By these means it is now possible to decide, to a greater or less degree of precision, the physiological needs of such divers individuals as pregnant women, adolescent children or coal-miners for calories, protein, fat, four or five mineral substances and six or seven vitamins. In making these advances in knowledge, the subjective feelings of the individuals concerned have not only been neglected; they have specifically been excluded. This has led to several curious conclusions, two at least of which may be cited.

There is, for example, no scientific evidence to suggest that violent muscular work has any influence whatever on the physiological demands of an individual for animal protein; yet there is a widespread popular belief that meat is essential for the efficient prosecution of manual labour. Similarly, so far as the classical nutritionist is aware, onions, garlic and pickles are of negligible nutritional value. Nevertheless, so pressing is the popular demand for such condiments that Britain, faced with extreme pressure on her land for food and aerodromes, is compelled to devote a substantial acreage to their culture.

Now, into the traditional, generation-old field of respectable nutrition comes Prof. Curt Richter, of the Johns Hopkins University School of Medicine*, with a summary of a number of inconvenient papers which have been appearing in the physiological literature during the last ten years, and the implications of which have largely been ignored by nutritionists.

Prof. Richter begins quietly. If rats are kept in a cage on a salt-free diet and given the opportunity of drinking a 3 per cent salt solution out of a graduated tube they will, on the average, drink enough of the

" "Total Self-regulatory Functions in Animals and Human Beings." Curt P. Richter. The Harvey Lectures Series, 38, 63 (1942-43). 